\title{
Surviving COVID-19: A disease tolerance perspective
}

\author{
Janelle S. Ayres \\ Molecular and Systems Physiology Laboratory, Gene Expression Laboratory, NOMIS Center for Immunology and Microbial Pathogenesis, The Salk Institute for Biological \\ Studies, 10010 North Torrey Pines Road, La Jolla, CA 92037 USA. Email: jayres@salk.edu
}

In December 2019, an epidemic of pneumonia of unknown cause emerged in Wuhan, China. In early January 2020, a virus was sequenced and identified as a novel coronavirus named SARS-CoV-2, the causative agent of COVID-19. By March 2020, the World Health Organization (WHO) declared the outbreak a pandemic with current numbers reaching over one million individuals infected and approximately 75,000 deaths globally. There has always been a disconnect between our methods for treating infectious diseases and our understanding of the mechanisms that promote the survival of infections (1). This global pandemic has underscored the necessity to understand how it is that we survive infections, and why this may be different from the ways we often think about the treatment of infectious diseases.

A successful response to any infectious disease outbreak requires a multipronged approach. With COVID-19, governments are enforcing unprecedented quarantines and social distancing measures to facilitate the containment and reduce transmission of the virus - efforts that are proving to be effective. Scientists from private and public sectors are racing to identify a successful vaccine, which will be essential for the prevention of future infections and mortalities, thereby reducing pressures on the healthcare system, economy and society. Where these efforts fall short are with the current approaches for developing treatments for those that are sick with and dying from a COVID-19 infection. The current potential COVID-19 treatments being tested include anti-virals already in use for HIV, anti-malarial drugs and other compounds that may prevent viral replication, and convalescent serum. The WHO has a similar focus on identification of therapies that destroy the virus with their launch of the SOLIDARITY trial that is streamlining the testing of multiple anti-viral strategies that may be effective against COVID-19 (2). The unifying goal for these therapeutic efforts is the identification of drugs that target the virus to inhibit viral replication.

Anti-virals will likely be effective for the fraction of infected patients that develop "mild" cases of COVID-19 by shortening their length of infection and for reducing transmission to naïve individuals. But for the patients who develop severe and critical disease, and who are destined for hospitalization and intensive care, the anti-viral based strategy does not jive with what is needed at the frontlines, where the medics and patients are fighting for life. These patients progress to severe and critical stages associated with pneumonia, acute respiratory distress syndrome (ARDS) and respiratory failure, septic shock and multi-organ dysfunction (3) - conditions that are caused by the host response to the virus. With these patients, the issue at hand is to sustain physiological function and buy them time so that they can get off the path to death and begin one toward recovery. In order to do this, doctors rely on supportive care such as mechanical ventilators, fluids, oxygen, blood pressure and anti-clotting medications - not anti-virals. The importance of identifying effective methods to target the host response to the infection rather than developing specific anti-virals for critically ill patients is underscored by clinical data involving patients with influenza. Approximately $25 \%$ of critically ill patients that receive optimal anti-viral therapy still die (4). This implicates that the host response to the virus is a major factor in determining the outcome of an influenza, and likely COVID-19 infections. So, while hospitals and governments race to find sufficient amounts of supportive care equipment as their healthcare systems are threatened with exceeding capacity, scientists are focused on developing anti-virals and not on drugs that promote physiological function during the infection. In addition to developing anti-virals, we need therapeutics that perform the functions of supportive care so that doctors are better equipped with an arsenal of therapies that can target any aspect of the patient's physiology to sustain its function. Such therapies will not only operate to promote survival, they also come with less risk of the pathogen developing drug resistance that will eventually happen with the anti-virals, because these strategies target the health of the host, and not the virus (5).

There is no scientific or public health reason for why we have not developed such therapeutics. It was described more than a decade ago that the infection defense response relies on essential mechanisms for survival that limit damage to the host and promote physiological function, rather than targeting the pathogen $(6,7)$. These mechanisms are called "disease tolerance" mechanisms encoded by the host's "cooperative defense system" and are essential for survival following infections and operate to achieve the same goal as supportive care. The cooperative defense system also encodes "anti-virulence" mechanisms that neutralize pathogen and host derived pathogenic signals that cause damage (8). There are considerations for anti-virulence-based strategies for COVID-19 treatments including blocking components of the innate immune system such as IL-6 and inflammasome activation. 
There are two potential concerns for these particular approaches. First, is that they can increase patient susceptibility to the virus or secondary bacterial infections because they block immune responses. Second, there is an important temporal aspect to the infection that needs to be considered. By the time a patient with severe respiratory viral infection presents for care and is hypoxic, the initiating pathogenic responses by the host that lead to ARDS have already occurred. Therefore, it's questionable whether focusing on the initial events leading to tissue damage make sense, and perhaps the more logical approach is to focus on the disease tolerance components of the host defense response that will sustain physiological function in the presence of this damage and that initiate a recovery response. From a public health perspective, it makes sense to develop host directed strategies to promote sustaining physiological function. For any given outbreak, we do not necessarily know the pathogen beforehand, and therefore likely will not be armed with effective vaccines and anti-microbials. We do, however, know how the body works and that despite the primary cause of disease, there are a finite number of ways in which a patient can develop pathology and die (8). We therefore can develop disease tolerance drugs that alleviate these pathologies, and/or promote physiological function in the face of these pathologies that we can call upon for the next pandemic, to reduce patient mortality, while we wait for effective vaccines and anti-microbialbased strategies.

The simple explanation for this disconnect is that the perspective for combating infectious diseases shared by scientists is incomplete. The fields of immunology and microbiology have focused on understanding strategies to kill the infection, which has provided us with some of the most important innovations for global health - vaccines and antimicrobials. But while this perspective is valuable, it is not enough. Instead of asking "how do we fight infections?" we might start asking "how do we survive infections?". To understand the answer to this question, we must approach infectious diseases at the molecular, cellular, organ, physiological and organismal levels. We have an understanding of the mechanisms of disease pathogenesis for COVID-19related pathologies and now we need to understand the mechanisms that restore normal function in the body and how we can drug these pathways for COVID-19 treatment. For example, understanding how lung function is normally restored when perturbed can inform us as to how it can be maintained in the face of infection. The complications associated with ARDS includes hypoxia due to damage of the alveolar epithelial and endothelial capillary barriers, leading to fluid accumulation, alveolar collapse and reduced gas exchange. Fluid reabsorption and surfactant production involve metabolic processes performed by the alveolar epithelial cells. Can we manipulate the metabolism of these cells to sustain surfactant production and secretion, and fluid reabsorption from the alveoli to promote gas exchange and prevent extra-pulmonary pathologies caused by respiratory failure? The alveolar epithelial and capillary endothelial barriers are disrupted by inflammatory signals of immune cells. Can we manipulate the physiology of the epithelial and endothelial cells so that they are resistant to the pathogenic signals and therefore maintain the barrier preventing fluid accumulation and poor gas exchange? Are there insights to be gleaned from the treatments of other lung diseases? Are there insights that can come from progress in wound repair, vascular function, and metabolic disease? Do the answers for disease tolerance mechanisms lie with those that are asymptomatic carriers of COVID-19 and those that are mildly symptomatic? Recruiting experts from diverse fields that cover all aspects of host and pathogen physiology will make us better equipped to deal with the complex nature of host survival of infections. Perhaps, by stepping beyond our focus on the virus, we may learn how to survive it.

\section{REFERENCES AND NOTES}

1. D. S. Schneider, J. S. Ayres, Two ways to survive infection: What resistance and tolerance can teach us about treating infectious diseases. Nat. Rev. Immunol. 8, 889-895 (2008). doi:10.1038/nri2432 Medline

2. K. Kupferschmidt, J. Cohen, Race to find COVID-19 treatments accelerates. Science 367, 1412-1413 (2020). doi:10.1126/science.367.6485.1412 Medline

3. H. K. Siddiqi, M.R. Mehra, COVID-19 illness in native and immunosuppressed stated: a clinical-therapeutic staging proposal. J. Heart Lung Transplant. (2020). doi:10.1016/j.healun.2020.03.012

4. J. K. Louie, S. Yang, M. Acosta, C. Yen, M. C. Samuel, R. Schechter, H. Guevara, T. M. Uyeki, Treatment with neuraminidase inhibitors for critically ill patients with influenza A (H1N1)pdm09. Clin. Infect. Dis. 55, 1198-1204 (2012). doi:10.1093/cid/cis636 Medline

5. J. S. Ayres, Cooperative Microbial Tolerance Behaviors in Host-Microbiota Mutualism, Cell 165, 1323-1331 (2016). doi:10.1016/j.cell.2016.05.049 Medline

6. J. S. Ayres, N. Freitag, D. S. Schneider, Identification of Drosophila mutants altering defense of and endurance to Listeria monocytogenes infection. Genetics 178 , 1807-1815 (2008). doi:10.1534/genetics.107.083782 Medline

7. L. Råberg, D. Sim, A. F. Read, Disentangling genetic variation for resistance and tolerance to infectious diseases in animals. Science 318, 812-814 (2007). doi:10.1126/science.1148526 Medline

8. J. S. Ayres, The biology of physiological health. Cell (2020). doi:10.1016/i.cell.2020.03.036

Published First Release 17 April 2020

10.1126/sciadv.abc1518 